\title{
Argumentos declamatorios y autonomía poética en la primera invectiva In Rufinum de Claudiano
}

\author{
Álvaro SÁNCHEZ-OsTiZ \\ Universidad de Navarra \\ asostiz@unav.es
}

Recibido: 12 de febrero de 2013

Aceptado: 4 de mayo de 2013

\section{RESUMEN}

Este estudio desarrolla un argumento adicional a favor de la discutida autonomía literaria de la primera invectiva In Rufinum de Claudio Claudiano. En esta composición el poeta alejandrino invirtió el argumento declamatorio que Juvenal había utilizado en su Sátira XIII para cohesionar la sucesión de cuadros. Algunos rasgos de la recepción de Juvenal en el ambiente intelectual romano del siglo IV posibilitan la referencia ejemplar que Claudiano hace al texto de las Sátiras. Esta hipótesis se corrobora asimismo mediante la comparación entre la alusión descrita y usos semejantes de hilos argumentales secundarios en un poema no invectivo, el Panegyricus dictus Mallio Theodoro consuli. Este tipo de imitación no satírica del modelo de Juvenal por parte de Claudiano, que no ha sido explorada hasta la fecha, permite precisar el modo en el que el poeta combina dinámicamente los géneros y se posiciona en la tradición literaria griega y romana.

Palabras clave: Claudiano. Juvenal. Panegírico. Invectiva. Sátira. Invectiva In Rufinum I.

SÁnCHEZ-OstiZ, A., «Argumentos declamatorios y autonomía poética en la primera invectiva In Rufinum de Claudiano», Cuad. Fil. Clás. Estud. Lat. 33.1 (2013) 57-72.

\section{Declamatory arguments and poetic autonomy in Claudian's first invective In Rufinum}

\begin{abstract}
This paper presents a new argument defending the long debated literary autonomy of Claudius Claudianus' first invective In Rufinum. In this work, the poet from Alexandria reversed the declamatory argument that Juvenal had deployed in his Satire XIII in order to reinforce the unity between the vignettes in his invective. Some features of Juvenal's reception in the Roman intellectual milieu of the 4th century enable to understand this archetypal reference Claudian makes to the satires. This hypothesis is supported by comparing the mentioned allusion with similar uses of such secondary argumentative threads in a non-invective poem of Claudian, the Panegyricus dictus Mallio Theodoro consuli. This non-satirical imitation of Juvenal by Claudianus, not explored so far, gives nuances to the mood in which the poet combines dynamically the genres placing himself in the Greek and Roman literary tradition.
\end{abstract}

Keywords: Claudian. Juvenal. Panegyric. Invective. Satire. Invective In Rufinum I.

SÁncheZ-Ostiz, A., «Declamatory arguments and poetic autonomy in Claudian's first invective In Rufinum», Cuad. Fil. Clás. Estud. Lat. 33.1 (2013) 57-72.

SUMARIO 1. Introducción. 2. Unidad y autonomía poética de los dos libros In Rufinum. 3. El modelo de la Satura 13 de Juvenal en Ruf. 1. 4. Hilos argumentales secundarios en el Panegírico al cónsul Manlio Teodoro. 5. Conclusiones. 6. Referencias bibliográficas. 


\section{INTRODUCCIÓN}

Las invectivas que Claudiano compuso contra Rufino y Eutropio ${ }^{1}$, regentes $d e$ facto en la corte de Constantinopla durante los años 396-399, han suscitado una atención particular en el debate sobre la naturaleza de los géneros literarios que el alejandrino combina en el conjunto de su poesía política ${ }^{2}$. Al igual que los panegíricos y epopeyas históricas del poeta, estos cuatro poemas entrelazan elementos de la tradición épica y de la oratoria epidíctica, pero además dan cabida a motivos inspirados en la sátira romana, especialmente en el modelo de Juvenal ${ }^{3}$. Como consecuencia, el In Rufinum (Ruf.) y el In Eutropium (Eutr.) presentan al lector ${ }^{4}$ un exigente juego de alusiones intertextuales y convenciones genéricas.

En este contexto, los poemas contra Rufino, transmitidos en dos libros por la tradición manuscrita, añaden dos puntos de discusión específicos. Por un lado, no resulta sencillo categorizarlos como meras invectivas, por cuanto sus secciones narrativas y abiertamente encomiásticas de Teodosio y de Estilicón adquieren mayor protagonismo funcional que las secciones que vituperan al prefecto del pretorio. Por otro lado, es asimismo controvertido si Claudiano proyectó ambos poemas como dos partes diferentes de una sola unidad literaria, o bien como dos unidades literarias autónomas que trataban un mismo tema, pero que fueron presentadas públicamente en dos ocasiones diferentes. Estas dos cuestiones trascienden el análisis meramente formal y comportan importantes implicaciones acerca del género de ambos poemas y de la controvertida cronología de la producción del alejandrino durante los años 396-397.

En este trabajo defenderé la autonomía literaria del primer libro del In Rufinum (Ruf.1), basándome en que Claudiano dio cohesión a las diferentes secciones de su poema invirtiendo un argumento declamatorio tradicional, tomado deliberadamente de la sátira 13 de Juvenal. Este análisis aportará a su vez algunas precisiones acerca del modo en que Claudiano amalgama los géneros literarios, concretamente cómo convierte su invectiva contra Rufino en un encomio anti-satírico de Honorio y Estilicón. Antes de desarrollar esta hipótesis, resulta pertinente recordar qué puntos esenciales han centrado la discusión sobre la unidad poética de los dos libros In Rufinum.

\footnotetext{
${ }^{1}$ Este trabajo se ha beneficiado del proyecto de investigación «Alteritas: Alteridad lingüística y alteridad cultural en el Imperio Romano (ss. III-V): historiografía y géneros afines» (Ref. FFI2010-15402/FILO) financiado por el Ministerio de Economía y Competitividad de España. Agradezco a J.B. Torres sus observaciones. Los errores e imprecisiones son atribuibles exclusivamente a mí.

${ }^{2}$ Determinar si la amalgama de épica, panegírico y otros géneros ha de denominarse «épica panegírica» (Hofmann 1988; Charlet 2000, pp.XXXVI-XL), «panegírico en verso» (Schindler 2004; 2009) o «poesía político-contemporánea» (Schmidt 1976; Müller 2011, passim, esp. pp.19-35) es cuestión casi proteica, pero no precisamente banal para entender las condiciones de creación y recepción en las que ha de entenderse la obra del alejandrino. El asunto es asimismo inseparable de la ya larga discusión sobre el carácter «propagandístico» de la producción claudiánea: véase sobre todo Cameron (1970); Gnilka (1977, pp.26-51); Döpp (1980); Moroni (1982). Asimismo, Cameron (2000) y Gnilka (2007) matizan sus respectivas posturas.

${ }^{3}$ Sobre todo a partir de Birt (1888, esp. pp.52-63). Los pormenores son discutidos por Long (1996a, pp.5163), Garambois-Vasquez (2007, pp.149-155) y Schmitz (2009).

${ }^{4}$ Los poemas tuvieron sin duda dos audiencias diferentes: la de su primera recitación en la corte de Milán y la que facilitó en un segundo momento la versión publicada (Cameron 1970, pp.228-252; Charlet 2009, pp.1-10).
} 


\section{UNIDAD Y AUTONOMÍA POÉTICA DE LOS DOS LIBROS IN RUFINUM}

Los dos poemas de Claudiano contra Flavio Rufino, prefecto del pretorio de Oriente (392-395) de origen galo, $R u f .1$ (c. 3) y Ruf.2 (c. 5), se consideraban ya desde antiguo una sola composición dividida en dos partes, puesto que la tradición manuscrita los transmite respectivamente como liber prior y liber alter. También se ha interpretado como indicio de unidad que la praefatio de Ruf.2 (c. 4), referida al contexto comunicativo de su presentación en público, se halla pospuesta a $R u f .2$ y antecede a Gild. en casi todos los manuscritos de la serie I. Esta dislocación indicaría que ambos poemas se recitaron conjuntamente en Milán después del verano del 397 ante el propio Estilicón, quien es apostrofado en los versos Ruf.2.13-16, una vez que éste hubiese regresado de su campaña contra Alarico en Grecia (Fargues 1933, p.15; Hall 1986, pp.70-71 $)$. Es decir, la sección que hoy se conoce como praefatio de $R u f .2$ (c. 6) sería una praefatio específica elaborada a posteriori para una segunda recitación oficial.

Asimismo, la indudable afinidad temática que tienen ambos libros aporta un ulterior indicio de unidad, reforzado por diversas correspondencias entre ambas partes: por un lado, en las escenas finales del primer libro (1.354-387) Justicia profetiza la inminente muerte deshonrosa de Rufino y el fausto advenimiento de una nueva Edad de Oro bajo el reinado de Honorio, mientras que el segundo relata los acontecimientos hasta ese desenlace en Constantinopla el 27 de noviembre del 395; por otro lado, podría entenderse que la laus Stilichonis sería el motivo principal de las secciones comprendidas entre los versos $R u f .1 .259$ y 2.256 , en el caso de que no hubiese solución de continuidad al final del primer libro. Para Schmidt (1976, pp.5962 , esp. p.60) ${ }^{6}$, tanto las continuidades como el final abrupto del liber prior indicarían que Claudiano diseñó un plan unitario para el conjunto a principios del 396, pero las noticias de los acontecimientos en Grecia le habrían sugerido modificar su programa, dejar inconcluso Ruf.1 y continuar con Ruf.2. En esta misma línea, Döpp (1980, pp.89-101; 2000) considera que el poeta habría compuesto consecutivamente y en ausencia de Estilicón Praef.Ruf.1 (c. 2), Ruf.1 (c. 3) y Ruf.2 (c. 5) como un solo proyecto literario entre el 396 y verano del 397, pero habría redactado Praef.Ruf.2 (c. 4) después del desenlace de la campaña contra Alarico. El conjunto combinaría así dos modos distintos de presentación, el modo invectivo del primer libro y el modo narrativo del segundo, con el fin de subrayar que Rufino era el responsable de la retirada de Estilicón en el 395 y de las incursiones godas en la Hélade durante los años 396-397.

Asimismo, los autores que han examinado la estructura retórica y los elementos

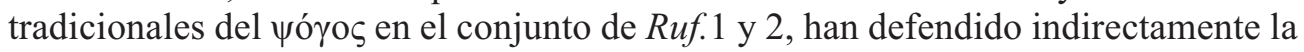
unidad de ambas partes. Según el análisis de Levy (1946, pp.57-65; 1971, esp. pp.256-

\footnotetext{
${ }^{5}$ Véanse, sin embargo, las matizaciones de Schmidt (1989, pp.403-409; 2004, pp.200-202) y Felgentreu (1999, pp.61-65).

${ }^{6}$ Asimismo Cameron (1970, p.79) considera también que Ruf.1 estaría inacabado, pero sería autónomo de Ruf.2.
} 
259) ${ }^{7}$, Claudiano habría aplicado sin rigidez las líneas maestras de la preceptiva al primer libro, siguiendo el esquema propio de un panegírico inverso. Así, después del

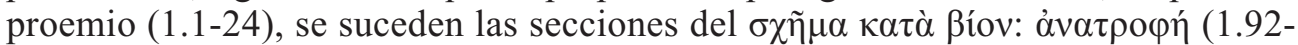

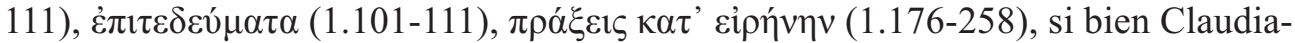

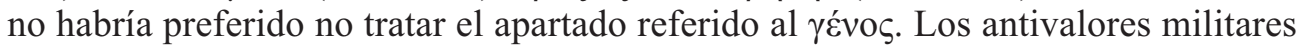

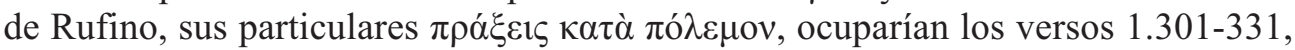
1.354-387, así como buena parte del libro 2 (2.1-383), hasta la escena final del lin-

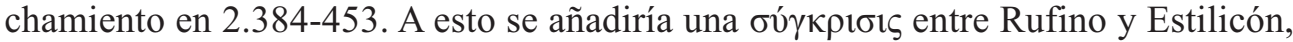
que se desarrolla como sección laudatoria de éste último (1.259-300) y que cohesionaría el conjunto ${ }^{8}$.

Por su parte, quienes han argumentado que cada poema fue una obra literaria autónoma, recitada en una ocasión diversa, se han basado en que los respectivos libros muestran significativas diferencias de género literario, de contenido y de estructura. En primer término, Cameron (1970, pp.76-90) destacó que Ruf.1 está plan-

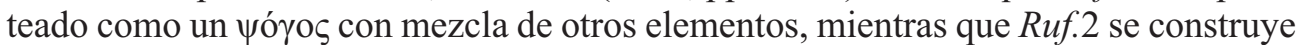
como un epos histórico de narración cronológica, por lo que cada parte respondería a un proyecto diferente (Felgentreu 1999, pp.67-76). El primero sería una invectiva escrita a comienzos del año 396, prácticamente a continuación del asesinato de Rufino, mientras que el segundo libro sería un relato presentado a finales del verano del 397, que pretende disimular las calamitosas operaciones de Estilicón en Grecia ese mismo año, dirigiendo la atención sobre el señuelo de los sucesos ya remotos ocurridos en el $395^{\circ}$.

En segundo término, aun concediendo que existen afinidades ya aludidas entre ambos libros, se ha destacado asimismo que el desarrollo del primero estaría dominado por la intervención de los dioses, mientras que el aparato divino se hallaría ausente en el segundo, lo que representa una discontinuidad notable entre ambos textos. El mensaje particular de Ruf.1 implica a fuerzas sobrehumanas enfrentadas entre sí, alineadas respectivamente con Rufino y con Teodosio y que funcionan como motor principal de la acción. Con esta estrategia argumental, el padre de Arcadio y Honorio quedaría descargado de la responsabilidad en el ascenso al poder de Rufino, puesto que habría sido víctima de una conspiración infernal (Müller 2011, pp.122, 137-143, 148-155).

\footnotetext{
${ }^{7}$ En esta misma línea inciden también Prenner (2001) y Koster (1980, pp.298-314). Gualandri (2002a, pp.53-74) parte también de la unidad de ambos poemas, al entender que In Rufinum 1 y 2 caracterizan al malvado utilizando ecos de la literatura apocalíptica del momento, en especial del Asclepius.

${ }^{8}$ Levy $(1946$; 1971) subraya, sin embargo, que el carácter épico-narrativo se impone al estrictamente invectivo; en este mismo sentido se pronuncian también Barr (1979) y Kirsch (1989, pp.159-160). Por su parte, Potz (1990) destaca igualmente que estas composiciones tienen como propósito literario principal la laudatio de Estilicón más que la invectiva de Rufino. Se pueden ver los pormenores de la discusión en Long (1996a, pp.44-48) y Prenner (2001).

${ }^{9}$ Ahora bien, aun concediendo que las diferencias formales entre ambos libros son notables, Ruf. 1 no constituiría una invectiva stricto sensu, sino una sucesión de escenas, algunas narrativas, otras descriptivas, con otros elementos insertados de carácter invectivo, y que además concede un espacio primordial al encomio de Honorio y Estilicón (Schmitz 2009, pp.193-198).
} 
En último término, Nesselrath (1991, pp.217-231) ${ }^{10}$ explicó además cómo la estructura de Ruf.1, siendo propiamente narrativa, se basa en una alternancia simétrica de las sucesivas escenas, que integra de manera coherente el final abierto. Efectivamente, existe una responsio entre las secciones del poema, que giran en torno a una parte central, la бó $\gamma \kappa \rho ı ı ı$ entre las actuaciones de Rufino en Constantinopla frente a las de Estilicón en campaña (1.260-334). En torno a este núcleo se sitúa un primer anillo concéntrico en el que por una parte Megera visita a Rufino para desencadenar la funesta actuación del regente (1.123-170) y por otra se presenta a Marte en compañía de Estilicón (1.334-353). En un segundo anillo, la asamblea del inframundo (1.25122) constituye un primer polo opuesto a la sección final con los discursos de Megera y Justicia (1.354-387). Por contraste, Ruf.2 prescinde de esquemas anulares y presenta un relato lineal de las acciones de Rufino hasta su muerte violenta (2.277-453) y su juicio en el inframundo (2.454-527). Todo ello permite suponer que Claudiano habría redactado Ruf.1 en los últimos meses del 395 al mismo tiempo que IIICons.Hon. con intención de recitarlos en el cambio de año 395/396 ${ }^{11}$.

Como ya he apuntado, el propósito de este trabajo es proporcionar un argumento adicional para entender que en $R u f .1$ se trata de una unidad poética autónoma. Concretamente atenderé al modo en el que este poema alude a un argumento retórico utilizado por Juvenal en su sátira 13 y lo invierte para dar énfasis y unidad a la estrategia expositiva de su primer poema contra Rufino. Por tanto, se analizará un tipo de emulación de Juvenal diferente de la imitación de motivos satíricos en sus invectivas, línea de estudio que ya ha sido, a mi juicio, suficientemente explorada (Long 1996a; 1996b; Garambois-Vasquez 2007; Colton 2010). La hipótesis que planteo puede corroborarse si se confronta con otros poemas en los que Claudiano se sirvió también de hilos argumentales secundarios para dar unidad a la estructura. Así pues, los apartados siguientes de este trabajo analizarán las coincidencias entre CLAUD.Ruf.1 y Juv.13 y las semejanzas estructurales entre $R u f .1$ y el panegírico consular a Manlio Teodoro.

\section{EL MODELO DE LA SATURA 13 DE JUVENAL EN RUF. 1}

La secuencia narrativa de Ruf.1 puede sintetizarse de la manera siguiente. El poeta plantea en una sección inicial (vv. 1-24) una duda ficticia sobre la existencia de la justicia y de una providencia divina que pueda ocuparse de castigar al malvado y de premiar al hombre virtuoso. En estos pocos versos se confrontan opiniones filosóficas estoicas (6-11) y epicúreas (16-19) sobre la noción de premio y castigo. No obstante, la cuestión queda pronto dilucidada en los últimos cinco versos de esta sección inicial (20-24):

\footnotetext{
${ }^{10}$ Asimismo Nesselrath (1994) destaca que Ruf.2, a diferencia de Ruf.1, muestra influencias de la sátira menipea.

${ }^{11}$ Funke (1984) argumenta asimismo que Ruf.1 habría sido compuesto todavía en vida de Rufino. En términos semejantes se expresan Perrelli (1992, pp.41-48) y Charlet (2000, pp.XXI-XXIV y L-LI).
} 
abstulit hunc tandem Rufini poena tumultum absoluitque deos. iam non ad culmina rerum iniustos creuisse queror; tolluntur in altum ut lapsu grauiore ruant. nos pandite uati, Pierides, quo tanta lues eruperit ortu.

A continuación, el poeta traslada la escena al inframundo, en el que la furia Alecto ha convocado un concilio de seres infernales (25-122) ya que el actual dominio de la Justicia en el mundo se le hace difícilmente soportable. Como resultado de la asamblea, Megera acude al lugar natal de su protegido Rufino, y consigue espolearlo para que el futuro prefecto marche a Constantinopla y dé rienda suelta a su singular maestría en las malas acciones (123-175). Entre éstas destacan la codicia y la crueldad (176-256), a las que sólo la actuación de Estilicón puede hacer frente (256-353). La acción se retoma con un discurso de Megera, que provoca a Justicia (354-367) quien por su parte profetizará la muerte violenta y deshonrosa de Rufino (369-379), así como el advenimiento de una nueva Edad de Oro bajo el reinado de Honorio (380$387)^{12}$. Las últimas palabras de Justicia suponen así una responsio a la cuestión planteada y cerrada en la sección inicial sobre el castigo de las acciones de los malvados. Sin embargo, el avance progresivo de las escenas e intervenciones constituye una presentación dramática de la controversia, que sirve de hilo argumental secundario en relación con el mensaje nuclear del poema, que es la oposición de las figuras de Rufino y Estilicón.

El debate sobre el castigo del malhechor desempeña asimismo un papel protagonista en la sátira 13 de Juvenal que construye su discurso como parodia de las consolaciones retóricas ${ }^{13}$. En efecto, el satírico —o la persona del satírico- brinda un repertorio de motivos parenéticos a su amigo Calvino por haber perdido diez mil sestercios, prestados a un tercero bajo juramento. La ira de su amigo sería excesiva, puesto que todos estamos sufriendo los mismos males (cf. 11-12: ponamus nimios gemitus. flagrantior aequo/ non debet dolor esse uiri nec uolnere maior). En la Edad de Oro los dioses eran pocos y la maldad un prodigio, en cambio ahora lo verdaderamente inaudito es que un amigo te devuelva el dinero prestado (vv. 38-70). Algunos no creen en los seres superiores y no esperan castigo, pero es innegable que a crímenes iguales siguen suertes bien distintas. Otros prefieren no devolver el dinero, puesto que los dioses no tendrán tiempo de perseguir a todos los criminales (71-125); la fechoría del dinero no devuelto no es tan grande comparada con los asesinatos y robos sacrílegos que sufren a diario los habitantes de Roma (126-173). El satírico acumula a partir de este punto argumentos para que su amigo deponga su airado deseo de castigo: la

\footnotetext{
${ }^{12}$ Los motivos pueden encontrarse en el repertorio habitual de los panegíricos de escuela, por ejemplo: $\mathrm{Pa}$ neg.10(1).11.3; 11(2).15.3-4; 9(4).18.1-5; 6(6).21.3-6; 5(7).13.1-3; 4(9).38.1-4; 3(10).10.1. Sobre el papel de la Edad de Oro en el imaginario poético de Claudiano, véase Charlet (2005).

${ }^{13}$ Adamietz (1984), Braund (1997b) y Keane (2007) sintetizan la bibliografía anterior sobre este punto. Sin embargo, Braund (1997b) matiza que la segunda parte del poema (Juv.13.174-249) no parodia motivos consolatorios, sino que adapta irónicamente el contenido de SEN.Dial.3.4.4 a propósito de la venganza y del castigo.
} 
venganza sería más propia de espíritus mezquinos (174-192); el peor escarmiento de los criminales es cargar en la conciencia con su propia culpa (192-239); además, su naturaleza empedernida les arrastrará a cometer nuevas fechorías que acabarán por ser castigadas con prisión, horca o destierro (239-249).

El satírico caricaturiza así motivos y textos de la literatura consolatoria sustituyendo el motivo más habitual de la muerte por el de la impunidad de determinados crímenes. Con ello el poema abunda en una recusatio de la filosofía, expresada más claramente por los versos de Juv.13.120-123, en los que Juvenal declara que no utilizará argumentos aprendidos en ninguna escuela de pensamiento: Accipe quae contra ualeat solacia ferre/ et qui nec Cynicos nec Stoica dogmata legit/ a Cynicis tunica distantia, non Epicurum/suspicit exigui laetum plantaribus horti. Este tipo de alusión al comienzo del libro $\mathrm{V}$ marca el tono de la nueva vena satírica que Juvenal va a explorar en las Sátiras 13-16 (Keane 2007), en cada una de las cuales retrata, tomando como punto de partida opiniones filosóficas conocidas, la degradación moral de Roma: ésta se encuentra no ya en una ferrea aetas, sino en una novena edad de decadencia progresiva (JUV.13.28-30): nona aetas agitur peioraque saecula ferri/ temporibus, quorum sceleri non inuenit ipsa/ nomen et a nullo posuit natura metallo.

Si bien las respectivas macro-estructuras de CLAUD.Ruf.1 y de JuV.13 divergen en aspectos esenciales, ambas presentan sin embargo significativos puntos de contacto. Al recomendar resignación ante la injusticia generalizada, Juvenal provoca en Calvino un enconamiento aún mayor en su peculiar enfado. Asimismo, la controversia sobre crimen y castigo acaba en un callejón sin salida (13.174-294), puesto que los malhechores llevan a cuestas el escarmiento en su mala conciencia, que sigue creciendo con la repetición de sus fechorías. Por su parte, la invectiva de Claudiano justifica rebelarse contra el tirano y darle muerte, como reparación de sus malas acciones. La divergencia de tono resulta especialmente significativa, puesto que el alejandrino excluye intencionalmente el matiz humorístico y todo rastro del ethos de indignación, característicos de Juvenal ${ }^{14}$. Es más, en abierto contraste con la Roma de Juvenal, esquilmada sin piedad por los robos sacrílegos y los rufianes sin escrúpulos, Claudiano despliega ante el lector la superioridad moral de la nueva Roma salvada por Estilicón, que goza de una rediviva Edad de Oro. De la comparación de elementos comunes tratados de manera precisamente opuesta se puede suponer que Claudiano contrapone ante el lector su argumento al de la consolación de Calvino.

Se puede objetar que las coincidencias de estrategia argumental entre ambos textos deben entenderse como manifestaciones independientes de un similar tono diatríbico. En efecto, las dos composiciones comparten algunas características de ese tipo de discurso pseudo-filosófico, en particular, el tono conversacional y la finalidad principalmente persuasiva, que se impone al tratamiento sistemático de la discusión (Witke 1970, pp.42-46 y 115; Koster 1980, p.305). En el caso de Juv.13, el tono conversacional queda marcado por la reiterada inserción de apóstrofes y refutaciones de la opinión contraria, mientras que el acercamiento no metódico al tema

\footnotetext{
${ }^{14}$ Sobre la indignación en el libro V de Juvenal: Braund (1997b); Keane (2007); contra Anderson (1982, pp.351-361).
} 
se refleja en el acopio de lugares comunes consolatorios. Éstos pertenecen en gran medida sin duda a la tradición parenética, pero se encuentran asimismo en el acervo de las declamaciones retóricas ${ }^{15}$. Por su parte, entre los elementos de diatriba en Ruf.1 destacan sobre todo las reflexiones de validez universal sobre la avaricia (semper inops quicumque cupit) y la Edad de Oro, presentes en los versos 196-219 (Koster 1980, pp.308-309; Schmitz 2009, pp.202-204). Éstas comienzan precisamente con un apóstrofe (quo, uaesane, ruis?) y permiten asimismo que el autor intervenga en primera persona (vv. 204-205: haec mihi paupertas opulentior, haec mihi tecta/ culmina maiora tuis).

No obstante, es importante subrayar que el discurso de la diatriba, en tanto que modo de presentar y de argumentar, no es en sí un género literario de límites precisos, sino un tipo de texto que puede convenir a unos géneros u otros, dependiendo de la estrategia argumental que adopte el autor. Formulado de otra manera, la diatriba constituye tan sólo la forma externa de estos dos mensajes y no su núcleo esencial (Anderson 1982, pp.250-251 y 430-431). Así, la sátira 13 de Juvenal combina la diatriba con argumentos y tonos propiamente retóricos, procedentes de los usos declamatorios, mientras que los elementos diatríbicos en la invectiva contra Rufino aporta notas auxiliares dentro de los tonos narrativos y epidícticos predominantes. En suma, si bien las semejanzas entre los dos textos analizados en estas páginas resultan notables, no se puede excluir sin embargo que ambos estén desarrollando de manera independiente un argumento común de carácter general — crimen y castigo, existencia de los dioses, decadencia moral de las diferentes edades_-, presente en la diatriba y en las controversias $^{16}$.

Ahora bien, el modo en el que Claudiano construye la sección inicial de Ruf.1 y plantea su duda retórica acerca de la existencia de los dioses sugiere que las coincidencias a las que he aludido son intencionales. En el amplio aparato de fuentes que incluye la edición de Birt, quien estaba convencido de que el género literario de $R u f .1$ era satírico ${ }^{17}$, se menciona únicamente un paralelo verbal entre JuV.13.86-88 y Ruf.1.14 , al que no se ha atribuido especial relevancia funcional ${ }^{18}$ :

JUV.13.86-88:

sunt in fortunae qui casibus omnia ponant et nullo credant mundum rectore moueri

\footnotetext{
${ }^{15}$ La influencia de la práctica declamatoria en Juvenal ha sido estudiada por extenso, entre otros por De Decker (1913), Kenney (1963), Anderson (1982, pp.297-298 y 414-80) y Braund (1997a).

${ }^{16}$ Así De Decker (1913, pp.44-50, 56-57 y 62-63). Véase, por ejemplo, el lugar común del conuicium temporum en SEN.Contr.1.pr.8-10; 2.pr.2; 2.1.1; 2.1.12; 2.2.7. En esta línea se expresan asimismo Charlet (2000, pp.186-187) y Guipponi-Gineste (2010, pp.146-147), que parten del supuesto de que Claudiano está retomando la cuestión desde un punto de vista filosófico, excluyendo la posibilidad de que se trate de una reutilización burlesca de material conocido.

${ }^{17}$ Birt (1888, pp.60-63; 1892, p.18) entiende que el modelo de la invectiva In Rufinum habría sido la sátira 10 de Juvenal, puesto que ambas desarrollan el motivo de la exaltación sin medida y la caída del tirano, en este caso la de Sejano. Véase a este propósito también Schmitz (2009, pp.211-213).

${ }^{18}$ No obstante, este evidente paralelo le permitió fijar el texto a Cameron (1968, pp.387-388). GaramboisVasquez (2007, pp.151-152) no considera que el pasaje aluda intencionalmente a Juvenal. Por su parte, Funke (1986, p.359) excluye un contexto satírico o invectivo del pasaje.
} 
natura uoluente uices et lucis et anni, atque ideo intrepidi quaecumque altaria tangunt.

Claud.Ruf.1.1-7:

Saepe mihi dubiam traxit sententia mentem, curarent superi terras an nullus inesset rector et incerto fluerent mortalia casu. nam cum dispositi quaesissem foedera mundi praescriptosque mari fines annique meatus et lucis noctisque uices, tunc omnia rebar consilio firmata dei, qui lege moueri sidera...

En buena lógica, un paralelo textual aislado, o una serie de ellos, no permitiría establecer conclusiones de dependencia literaria entre textos. No obstante, las coincidencias literales entre estos dos pasajes (nullus rector, casus, moueo, uices, lucis...), así como las ligeras modificaciones que introduce Claudiano (omnia casibus ponant omnia rebar consilio firmata dei; mundum moueri $\sim$ sidera moueri...), pueden entenderse en efecto como marcadores intertextuales para el lector, al que se le ofrece una clave de lectura para el resto de la invectiva ${ }^{19}$. No se trata sólo de que el poeta de Alejandría haya modelado estos hexámetros iniciales utilizando el lenguaje del satírico $^{20}$, sino que la evocación del texto concreto en la sección programática permite entender el conjunto, incluido el final aparentemente abierto, como una unidad. Los versos de Juv.13.86-88 describen la posición irreligiosa de los epicúreos respecto al castigo de la injusticia, como coartada para la impunidad de las fechorías en la Roma que él vive. Por su parte, Claudiano recoge el reto que plantea esta objeción y la soluciona ya en la sección inicial de $R u f$.1, pero retoma asimismo la idea con la descripción de la Edad de Oro en los versos finales (1.380-387).

Puede parecer obvio que este tipo de intertextualidad implique que tanto el panegirista como su público estuvieran familiarizados con el modelo más allá de lecturas sectoriales de pasajes aislados. Es conocido que en la segunda mitad del siglo IV, después de dos siglos de olvido, Juvenal volvía a gozar de éxito literario en los círculos intelectuales ${ }^{21}$, así como en otros entornos menos exigentes ${ }^{22}$. No obstante, en lo que atañe al presente análisis, la alusión que Claudiano hace en Ruf.1 al argumento de

\footnotetext{
${ }^{19}$ Claudiano evita las citas literales y modifica el orden de los elementos del original para marcar que su alusión contiene un mensaje preciso (Gualandri 1968; 2004, pp.78-95; Keudel 1970, pp.157-159).

${ }^{20}$ Combinándola con reminiscencias de Virgilio, Lucano, Juvenal e incluso Lucrecio (Cameron 1968, pp.387388; 1970, pp.327-331; Levy 1971, pp.9-18; Funke 1984).

${ }^{21}$ Cameron (1984; 2004, sobre todo pp.349-354; 2011, pp.410-420). El único papiro de Juvenal conservado, fechable en torno al año 500 y procedente de Antínoe, contiene escolios y glosas latinos y griegos, así como signos diacríticos, pertenecientes a varias manos, algunas de ellas griegas: $M-P^{3} 2925$. Por tanto, testimonia que a finales del siglo V Juvenal era utilizado para aprender latín en algunos lugares de habla griega.

${ }^{22}$ A éstos debían de pertenecer los jóvenes romanos sin gusto ni afición literaria, atraídos sólo por las sátiras del poeta de Aquinum y por las biografías de Mario Máximo, a los que se refiere Amiano: AmM.28.4.14: Quidam detestantes ut uenena doctrinas Iuuenalem et Marium Maximum curatiore studio legunt, nulla uolumina praeter haec in profundo otio contrectantes...
} 
JuV.13 supone además que al menos en los años en que Claudiano llega a Roma o inmediatamente antes era usual leer las sátiras del poeta de Aquinum como unidades literarias completas dentro de sus respectivos libros, puesto que la alusión se construye tanto en las coincidencias intertextuales, como en el nivel macroestructural de los argumentos.

Por último, es pertinente destacar que en estricto sentido el motivo declamatorio de la Edad de Oro posee una funcionalidad de segundo orden en Ruf.1. El poema es a to-

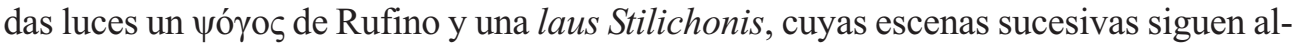
ternativamente un esquema narrativo y uno invectivo, pero al que se añade el contraste con motivo del castigo y la degeneración de las edades tomado de JuV.13. Ahora bien, aun concediendo que la función atribuida a este hilo dentro de conjunto no es principal, el hecho de que las coincidencias con JuV.13 se sitúen en los versos iniciales y finales precisamente subraya la unidad y autonomía de $R u f$. 1 como proyecto literario. Este hecho es relevante para la cuestión sobre los géneros de la poesía política de Claudiano, en tanto que esta invectiva-panegírico se construye en forma de anti-sátira, al revertir su modelo. Expresado de otro modo, el argumento del castigo del malvado en la degradación de las edades fue en su origen material retórico-declamatorio, presentado en Juvenal con el envoltorio formal de la diatriba; Claudiano lo retoma, en cambio, manteniendo rasgos del discurso conversacional, pero subrayando que sus héroes han traído una nueva edad que no deja lugar a la indignación satírica.

\section{HILOS ARGUMENTALES SECUNDARIOS EN EL PANEGÍRICO AL CÓNSUL MANLIO TEODORO}

Es interesante destacar, por último, que este recurso retórico no es un procedimiento aislado en la poética de Claudiano, quien utiliza técnicas semejantes en otras composiciones, lo cual apoya, a mi entender, la idea de que el análisis del comienzo de $R u f .1$ que propongo no es una sobreinterpretación del texto de esta invectiva. Así, las similitudes, entre Ruf.1 y el Panegírico al cónsul Manlio Teodoro (Theod.) aportan de hecho una evidencia intratextual al argumento de este trabajo. Se ha destacado con frecuencia que el alejandrino tiende a plantear sucesiones de cuadros descriptivos sacrificando un desarrollo estrictamente argumental del relato (Gualandri 1968, pp.8-9; 1998; Cameron 1970, pp.262-266; Fo 1982, pp.22-24; Gnilka 2007, pp.142-144). Cada cuadro o viñeta presenta una escena, realista o fantasiosa, en la que toman la palabra protagonistas o divinidades implicados en el mensaje político. Las transiciones entre las distintas escenas son en ocasiones marcadamente abruptas y quedan definidas por fórmulas introductorias del tipo est locus, o bien son anticipadas por el viaje de una divinidad desde su ámbito propio ante la presencia de uno de los personajes ${ }^{23}$. En otras ocasiones en cambio, el hilo retrocede tempo-

\footnotetext{
${ }^{23}$ Sin pretensión de exhaustividad, se puede mencionar aquí que en CLAUDIAN.Epit.228-294 Venus viaja desde Chipre hasta Liguria, para encontrarse con María y adornarla como novia. De manera semejante, en Stil.2.269-339 la diosa Roma ruega a Estilicón que asuma el consulado y en 408-476 el vuelo de la
} 
ralmente y la escena se traslada a un momento de singular importancia para el desarrollo de los acontecimientos, por ejemplo en IVCons.Hon.153-170; 214-352 y VICons.Hon.146-192.

Como se ha mencionado, este esquema estructura las primeras secciones de $R u f .1$, en las que la extensa queja de Alecto sobre la nueva Edad de Oro (1.45-65) es replicada por Megera (1.86-115), quien a su vez, disfrazada de anciana, viaja hasta la Galia para instigar las fechorías de Rufino (1.123-171). Ambas escenas fantásticas, la asamblea infernal y la singular epifanía de Megera ante Rufino, tienen lugar años antes de los sucesos que ocupan las secciones narrativas. Como he apuntado, el hilo secundario que resuelve la duda ficticia sobre la existencia de los dioses que habrán de castigar al malvado aporta unidad a la sucesión de cuadros.

Por su parte, el Panegírico al cónsul Manlio Teodoro presenta asimismo la misma combinación de intervenciones de los protagonistas enmarcadas en viñetas, cuya serie es cohesionada por un hilo secundario. Este poema difiere en sustancia de la primer invectiva contra Rufino, no sólo por su carácter abiertamente encomiástico, sino también porque su mensaje político está —al menos en apariencia - menos acentuado (Müller 2011, pp.218-222). Como panegírico, pronunciado a comienzos del año 399 con ocasión del inicio del año consular de Teodoro, subraya en primer término cómo el homenajeado fue aprendiendo el arte de gobernar durante sus primeros años, del mismo modo que un buen marinero llega a convertirse en el timonel de la nave (Theod.15-60). A continuación se destacan de manera pormenorizada los logros literarios y filosóficos del nuevo cónsul (Theod.61-113) ${ }^{24}$, quien después de retirarse por completo de la escena pública en el año 382 para dedicarse al ocio intelectual, había aceptado en el 397 la prefectura del Ilírico, Italia y África.

De manera semejante a $R u f .1$, la estructura de Theod. está basada en una serie alternante de escenas dispuestas en torno a una acción central, que se remonta unos cuantos años atrás: el viaje de Justicia (Theod.113-134) y su encuentro con Teodoro (Theod.135-197). Como consecuencia de la intervención de la diosa, Teodoro acepta abandonar su retiro intelectual y aceptar el cargo de prefecto del pretorio, cargo en el que realizará grandes acciones que son descritas en una amplia sección laudatoria (Theod.198269). Por último, el poema termina con un abrupto cambio de escenario desde Milán al Helicón (Theod.270-340). En este contexto fantástico, Urania, musa de la astronomía, toma la palabra invitando a sus compañeras a que participen en los juegos escénicos inaugurales del nuevo año y auspiciando que los descendientes de Teodoro seguirán siendo distinguidos con la trábea consular. Ha sido destacado ya oportunamente ${ }^{25}$

\footnotetext{
Fama prepara la sección final que describe la morada del Sol. En Stil.3.237-369 Diana recorre el mundo con su séquito en busca de fieras para los juegos consulares. Asimismo en Eutr.1.391-513 Roma ruega a Honorio y Estilicón que eliminen al chambelán; en Eutr.2.534-602 es Aurora quien pide a Estilicón protección para Occidente.

${ }^{24}$ Claudiano presentó este panegírico consular a comienzos del año 399, probablemente pocas semanas antes de su dura invectiva In Eutropium que marca un llamativo contraste en tono y tema.

${ }^{25}$ Gualandri (2002b). Es significativo que el De beata uita agustiniano (Aug.Beat.Vit. 1.2) esté dedicado a Teodoro en el año 386, utilizando precisamente la alegoría del viaje y del puerto de la filosofía en el proceloso piélago de la vida, lo que apunta a que ambos autores, refiriéndose a dos momentos diferentes de la vida de Teodoro, estaban rindiendo tributo a alguno de los temas filosóficos privilegiados por la obra filosófica de éste.
} 
que los motivos alegóricos de la navegación, dispersos a lo largo del poema, cohesionan la secuencia de escenas en una doble clave. Claudiano alude por una parte a la navegación personal del protagonista, que en medio de las tormentas de la existencia alcanza el puerto seguro de la filosofía ( $c f$. Theod.61-62: Postquam parta quies et summum nacta cacumen/ iam secura petit priuatum gloria portum ...), pero por otra parte también el poeta describe la progresión de la experiencia política de Teodoro, quien desde simple marinero ha ido aprendiendo el oficio hasta gobernar la nave entera de la res publica (cf. Theod.42-46, esp. 46: ... iam clauum totamque subit torquere carinam).

La alegoría de la navegación es lugar común en la tradición clásica y en la poesía latina anterior, por lo que la búsqueda de modelos concretos y de marcadores intertextuales en los versos de Claudiano podría parecer empresa irrealizable ${ }^{26}$. Ahora bien, a mi juicio el alejandrino completa el sentido del tópico con referencias a Cicerón ${ }^{27}$, implícitas en diferentes momentos del poema.

En este sentido se podría interpretar la insistencia de los versos de Theod. en la compatibilidad entre ocio literario y actividad política que ha mostrado el protagonista en su vida hasta el momento del consulado, ideales que sin duda eran del agrado de los sectores más tradicionalistas del senado. Pero el poeta establece además una comparación particular de contenido y forma entre la obra filosófica de Teodoro y los diálogos ciceronianos, puesto que el nuevo cónsul ha trasladado al latín las difíciles doctrinas de la filosofía griega utilizando el género de la disputatio in utramque partem (cf. Theod.84-86: Graiorum obscuras Romanis floribus artes/ inradias, uicibus gratis formare loquentes/ suetus et alterno uerum contexere nodo). La alusión es sin duda tácita y en estricto sentido discutible. Ahora bien, el lector culto pondría el extenso discurso de la musa Urania al final del panegírico (Theod.276-340), que concluye precisamente llamando a Teodoro grauissimus auctor eloquii en 333$334^{28}$, en relación con el que la misma musa pronuncia en el De consulatu suo de Cicerón (CIC.Div.1.17-22). Todo ello imbrica la sucesión en apariencia casual de viñetas en torno a la insinuación de que Teodoro habría sido para Roma un nuevo Cicerón.

En definitiva, el cambio de escenario en la parte central del panegírico de Manlio Teodoro presenta también, al igual que en $R u f .1$, un viaje espacio-temporal que concluye en un encuentro singular entre una divinidad (Justicia, Alecto) y el personaje protagonista (Teodoro, Rufino) ${ }^{29}$. Ambos encuentros resultan lejanos en el tiempo, pero mar-

\footnotetext{
${ }^{26}$ Nótese, por ejemplo, el paralelo de STAT.Theb. 10.180-184 con CLAUD.Theod. 42-46.

${ }^{27}$ Quien utiliza con frecuencia el repertorio de motivos náuticos para referirse a la existencia y a la política, véase entre otros pasajes, Cic.Cato 17; Div.2.3; Off.1.25.87; Phil.2.113; Pis.20; Rep.1.11.51; 2.3; S.Rosc.51; Sest.20; 27; 46; 99.

${ }^{28}$ Cicerón es llamado Romani maximus auctor eloquii por LUCAN.7.62-63, versos que el obispo de Hipona cita o parafrasea en Aug.Epist.143.3; 258.1; Doctr.Christ.4.17; Civ.14.18.

${ }^{29}$ Este esquema narrativo es similar, salvadas las distancias, al que rige en el Panegírico al cuarto consulado del Emperador Honorio. En éste la escena central (214-352) no es la visita de una divinidad al protagonista en el pasado, sino el encuentro entre Honorio niño y su padre Teodosio en el que éste le aconseja detalladamente sobre el modo de gobernar. Asimismo el panegírico concluye con un final breve pero abierto en alusión a la futura esposa del joven cónsul. La intervención de tramas o hilos secundarios en este panegírico, llamativamente extenso en comparación con otras obras de Claudiano, merece mayor atención que la que se puede dar dentro de los límites de este trabajo. Sobre la estructura de IVCons.Hon., véase Barr (1981, pp.20-24).
} 
carán el devenir posterior de los acontecimientos. En el primer caso, Justicia interviene provocando que Teodoro interrumpa su ocio filosófico y asuma el cargo de prefecto del pretorio (Theod.174-197). Ello antecede a su ascenso al consulado y le permite participar de la Edad de Oro propiciada por Estilicón (Theod.256-269). En el segundo caso, Alecto despierta a Rufino a una vida de rapiña, desenfreno y crueldad ${ }^{30}$. Los dos poemas además tienen un final engañosamente abierto que se revela sin embargo compatible con una estructura unitaria: mientras que en $R u f$.1.357-387 el discurso de Megera era contestado por Justicia, augurando una nueva aurea aetas, por su parte en una extensa écfrasis al final de Theod.270-340, Urania despliega ante las demás Musas los próximos juegos consulares. Es decir, en ambas composiciones Claudiano sitúa escenas variadas en torno a una sección central y las combina con un motivo argumental secundario: en un caso es el argumento retórico tomado de JuV.13 y en otro caso se trata de la metáfora de la navegación vital, perfilada con la comparación entre Teodoro con Cicerón.

\section{CONCLUSIONES}

De lo expuesto se concluye que Claudiano se sirvió del argumento declamatorio principal de JuV.13 para cohesionar la sucesión de escenas del Ruf.1, llevando a cabo con ello un original modo de emulación. Efectivamente, el castigo del malvado, avaro por más señas, en una nueva Edad de Oro refuerza de manera secundaria la unidad de las escenas dispuestas en una estructura concéntrica en torno a la sýncrisis entre Rufino y Estilicón. Esta evidencia apoya la opinión de que Ruf.1, por su propia unidad, ha de entenderse de manera autónoma respecto a $R u f .2$, como ejemplo de poesía política con un mensaje preciso: legitimar la muerte violenta de Rufino y marcar el contraste con el papel salvífico de Estilicón, cuya campaña contra Alarico en Grecia durante el 395 había sido especialmente cuestionable. Es posible asimismo que el poema fuese presentado a comienzos del 396, pero escrito poco antes de que hubiera llegado la noticia de la muerte del prefecto del pretorio de Oriente.

Sin embargo, Ruf.1 es una obra autónoma también con respecto al contexto comunicativo particular en el que fue presentada en público por primera vez. Es decir, el análisis desarrollado en este trabajo permite apreciar que el valor artístico de Ruf.1, en tanto que obra literaria conseguida, trasciende lo ocasional. Claudiano despliega ante el lector un retablo de maravillas: escenas y personajes del inframundo, la aparición de un vástago de los infiernos y el conveniente auxilio de Estilicón, garante a su vez de una nueva aurea aetas que, en la persona de Honorio, han previsto para Roma los dioses superiores. Las bruscas transiciones entre las viñetas provocan que los oyentes o lectores vayan de sorpresa en sorpresa. Por eso, quienes siguiendo la moda del momento conocían a fondo las sátiras de Juvenal percibían en Ruf.1 un juego de géneros literarios que engañaba sus expectativas. El discurso poético contra Rufino, una ar-

\footnotetext{
${ }^{30}$ Cf. Claud.Ruf.1.140: Otia te, Rufine, iunant frustraque iunentae/ consumis florem patriis inglorius aruis? y Theod.113-115: Iam tibi compositam fundauerat ancora puppim,/ telluris iam certus eras; fecunda placebant/ otia; nascentes ibant in saecula libri...
} 
madura invectiva montada sobre el bastidor de un poema épico narrativo, acababa convirtiéndose a sus oídos en una anti-sátira, un Juvenal domado por los guardianes de la nueva edad para alabanza de Honorio y Estilicón. La idea les resultaría hiperbólica, tanto más cuanto que esperarían que los tonos de la sátira, el género poético más genuinamente romano, acabasen permeando por mera afinidad un texto invectivo.

Con todo ello, la particular técnica de emulación descrita en estas páginas añade una nueva pieza a nuestro conocimiento del modo en que Claudiano entra en diálogo con los géneros literarios grecolatinos. En efecto, $R u f .1$ se presenta como inextricable mezcla de $\psi o ́$ os, de panegírico en prosa y de épica histórica en hexámetros, pero añade a ella una inversión del modelo de Juvenal y, por consiguiente, de la tradición satírica romana. Tal subversión no está exenta a su vez de ambigüedad: el griego Claudiano, virtuoso de la poesía en latín, no se contenta con aludir a Juvenal, sino que se muestra dispuesto a superarlo sin profundizar en la indignación satírica, es más, refutando los motivos de ésta.

\section{REFERENCIAS BIBLIOGRÁFICAS}

Adamietz, J. (1984), «Juvenals 13. Gedicht als Satire», Hermes 112, pp.469-483.

Anderson, W.S. (1982), Essays on Roman Satire, Princeton, Princeton University Press.

BARR, W. (1979), «Claudian's In Rufinum: an Invective?», PLLS 2, pp.179-190.

BARR, W. (1981), Claudian's Panegyric on the Fourth Consulate of Honorius, Liverpool, Cairns.

BIRT, T. (1888), Zwei politische Satiren des alten Rom, Marburgo, Elwert.

BIRT, T. (1892), Claudii Claudiani carmina, Berlín, Weidmann.

Braund, S.M. (1997a), «Declamation and Contestation in Satire», en Dominik, W.J. (ed.), Roman Eloquence: Rhetoric in Society and Literature, Londres, Routledge, pp.147-165.

Braund, S.M (1997b), «A Passion Unconsoled? Grief and Anger in Juvenal Satire 13», en Braund, S.M. y Gill, C. (ed.), The Passions in Roman Thought and Literature, Cambridge, Cambridge University Press, pp.68-88.

Cameron, A. (1968), «Notes on Claudian's Invectives», CQ 18, pp.387-411.

Cameron, A. (1970), Claudian: Poetry and Propaganda at the Court of Honorius, Oxford, Clarendon Press.

Cameron, A. (1984), «The Latin Revival of the Fourth Century», en Treadgold, W. (ed.), Renaissances before the Renaissance. Cultural Revivals of Late Antiquity and the Middle Ages, Stanford, Stanford University Press, pp.43-58.

CAmeron, A. (2000), «Claudian Revisited», en Consolino, F.E. (ed.), Letteratura e propaganda nell'Occidente latino da Augusto ai regni romanobarbarici, Roma, L'Erma di Bretschneider, pp.127-144.

CAmeron, A. (2004), «Poetry and Literary Culture in Late Antiquity», en SwaIn, S. y EDwArds, M.J. (ed.), Approaching Late Antiquity: the Transformation from Early to Late Empire, Oxford, Oxford University Press, pp.327-354.

CAmeron, A. (2011), The Last Pagans of Rome, Oxford, Oxford University Press.

Charlet, J.-L. (2000), Claudien. Oeuvres. II, 1: Poèmes politiques (395-398), París, Les BellesLettres. 
Álvaro Sánchez-Ostiz Argumentos declamatorios y autonomía poética en la primera invectiva In Rufinum de Claudiano

Charlet, J.-L. (2005), «L'age d'ôr dans la poésie de Claudien», en LehmanN, Y., Freyburger, G. y Hirstein, J. (ed.), Antiquité tardive et humanisme: de Tertullien à Beatus Rhenanus: mélanges offerts à François Heim à l'occasion de son 70e anniversaire, Turnhout, Brepols, pp.197-208.

Charlet, J.-L. (2009), «Claudien et son public», en Harich-SchwarzBauer, H. y Schierl, P. (ed.), Lateinische Poesie der Spätantike. Internationale Tagung in Castelen bei Augst, 11.-13. Oktober 2007, Basilea, Schwabe, pp.1-10.

Colton, R.E. (2010), «Echoes of Juvenal in Claudian's In Eutropium», en Deroux, C. (ed.), Studies in Latin Literature and Roman History. XV, Bruselas, Latomus, pp.492-514.

De DeCKer, J. (1913), Juvenalis declamans. Étude sur la rhétorique déclamatoire dans les satires de Juvénal, Gante, E. Van Goethem.

Döpp, S. (1980), Zeitgeschichte in Dichtungen Claudians, Wiesbaden, Steiner.

DöPP, S. (2000), «Der Sturz der Mächtigen in Claudians Invektive gegen Rufin», en WoLPERS, T. (ed.), Der Sturz der Mächtigen. Zu Struktur, Funktion und Geschichte eines literarischen Motivs, Gotinga, Vandenhoeck und Ruprecht, pp.73-94.

Fargues, P. (1933), Claudien. Etudes sur sa poésie et son temps, París, Hachette.

Felgentreu, F. (1999), Claudians praefationes: Bedingungen, Beschreibungen und Wirkungen einer poetischen Kleinform, Stuttgart, Teubner.

Fo, A. (1982), Studi sulla tecnica poetica di Claudiano, Catania, Tringale.

FunKe, H. (1984), «Zu Claudians Invektive gegen Rufin», ICS 9, pp.91-109.

FunKe, H. (1986), «The Universe of Claudian. Its Greek Sources», PLLS 5, pp.357-366.

GARAmBoIs-VASQUEZ, F. (2007), Les invectives de Claudien. Une poétique de la violence, Bruselas, Latomus.

GNILKA, C. (1973), «Götter und Dämonen in den Gedichten Claudians», $A \& A$ 18, pp.144-160.

GNILKA, C. (1977), Rec. de CAMERON 1970, Gnomon 49, pp.26-51.

GNILKA, C. (2007), Philologische Streifzüge durch die römische Dichtung, Basilea, Schwabe.

Gualandri, I. (1968), Aspetti della tecnica compositiva in Claudiano, Milán, Cisalpino.

GuAlandRI, I. (1998), «La poesia di Claudiano tra mito e storia», en Cultura latina pagana fra terzo e quinto secolo dopo Cristo, Atti del Convegno, Mantova 9-11 ottobre 1995, Florencia, Olschki, pp.113-143.

GuAlandRI, I. (2002a), «Echi apocalittici nell'In Rufinum di Claudiano», en GuALANDRI, I. (ed.), Tra IV e V secolo. Studi sulla cultura latina tardoantica, Milán, Cisalpino, pp.53-74.

Gualandri, I. (2002b), «Tra Agostino e Claudiano: riflessioni su Manlio Teodoro», en Isola, A., Menestò, E. y Pilla, A.D. (ed.), Curiositas. Studi di cultura classica e medievale in onore di U.Pizzani, Nápoles, Edizioni Scientifiche Italiane, pp.329-345.

Gualandri, I. (2004), «Claudian’s Greek World: Callimachus», en Ehlers, W.W., Felgentreu, F. y WhEELER, S.M. (ed.), Aetas Claudianea: Eine Tagung an der Freien Universität Berlin vom 28. bis 30. Juni 2002, Múnich, Saur, pp.78-95.

GuIPPONI-GineSte, M.-F. (2010), Claudien: poète du monde à la cour d'Occident, París, De Boccard.

Hall, J.B. (1986), Prolegomena to Claudian, Londres, University of London, Institute of Classical Studies.

HoFmANN, H. (1988), «Überlegungen zu einer Theorie der nichtchristlichen Epik der lateinischen Spätantike», Philologus 132, pp.101-159.

Keane, C. (2007), «Philosophy into Satire: The Program of Juvenal's Fifth Book», AJPh 128, pp.27-57. 
Álvaro Sánchez-Ostiz Argumentos declamatorios y autonomía poética en la primera invectiva In Rufinum de Claudiano

KenNEY, E.J. (1963), «Juvenal: Satirist or Rhetorician?», Latomus 22, pp.704-720.

Keudel, U. (1970), Poetische Vorläufer und Vorbilder in Claudians De consulatu Stilichonis: Imitationskommentar, Gotinga, Vandenhoeck und Ruprecht.

KIRSCH, W. (1989), Die lateinische Versepik des 4. Jahrhunderts, Berlín, Akademie-Verlag.

Koster, S. (1980), Die Invektive in der griechischen und römischen Literatur, Meisenheim am Glan, Hain.

LEVY, H.L. (1946), «Claudian's In Rufinum and the Rhetorical Yó

Levy, H.L. (1971), Claudian's In Rufinum: an Exegetical Commentary, Cleveland, Case Western Reserve University Press.

Long, J. (1996a), Claudian's In Eutropium, or, How, when, and why to Slander a Eunuch, Chapel Hill, University of North Carolina Press.

LoNG, J. (1996b), «Juvenal Renewed in Claudian's In Eutropium», IJCT 2, pp.321-335.

Moroni, B. (1982), «Tradizione letteraria e propaganda: Osservazioni sulla poesia politica di Claudiano», Scripta Philologica 3, pp.213-239.

Müller, G.M. (2011), Lectiones Claudianeae: Studien zu Poetik und Funktion der politischzeitgeschichtlichen Dichtungen Claudians, Heidelberg, Winter.

Nesselrath, H.-G. (1991), «Zu Datierung und Aufbau des I. Buches von Claudians Invektive 'In Rufinum'», Hermes 119, pp.217-231.

Nesselrath, H.-G. (1994), «Menippeisches in der Spätantike: Von Lukian zu Julians 'Caesares' und zu Claudians 'In Rufinum'», $M H$ 51, pp.30-44.

Perrelli, R. (1992), I proemî claudianei tra epica ed epidittica, Catania, Centro di studi sull'antico cristianesimo, Università di Catania.

Potz, E. (1990), «Claudians In Rufinum. Invektive und Laudatio», Philologus 134, pp.66-81.

PRENNER, A. (2001), «I 'topoi’ encomiastici nell'invettiva di Claudiano contro Rufino», Kolvwvía 25, pp.45-57.

SCHINDLER, C. (2004), «Tradition - Transformation - Innovation: Claudians Panegyriken und das Epos», en Ehlers, W.W., Felgentreu, F. y Wheeler, S.M. (ed.), Aetas Claudianea: Eine Tagung an der Freien Universität Berlin vom 28. bis 30. Juni 2002, Múnich, Saur, pp.16-37.

SCHINDleR, C. (2009), Per carmina laudes: Untersuchungen zur spätantiken Verspanegyrik von Claudian bis Coripp, Berlín, Walter De Gruyter.

ScHMidT, P.L. (1976), Politik und Dichtung in der Panegyrik Claudians, Constanza, Universitätsverlag.

Schmid, P.L. (1989), «Die Überlieferungsgeschichte von Claudians Carmina maiora», ICS 14, pp.391-415.

SCHMIDT, P.L. (2004), «Rezeptionsgeschichtliche Erwägungen zur Claudianüberlieferung», en Ehlers, W.-W., Felgentreu, F. y Wheeler, S. (ed.), Aetas Claudianea: eine Tagung an der Freien Universität Berlin vom 28. bis 30. Juni 2002, Múnich, Saur, pp.187-206.

Schmitz, C. (2009), «Satire / Invektive und Panegyrik in Claudians politischen Epen», en Felgentreu, F., Mundt, F. y Rücker, N. (ed.), Per attentam Caesaris aurem: Satire die unpolitische Gattung?: eine internationale Tagung an der Freien Universität Berlin vom 7. bis 8. März 2008, Tubinga, Narr, pp.192-227.

WitKe, C. (1970), Latin Satire: the Structure of Persuasion, Leiden, Brill. 